\title{
Traducción y recepción del 'Quijote' en Gran Bretaña (1612-1774)
}

\begin{abstract}
J.A.G. ARDILA*
La mayoría de los ensayos contemporáneos que consideran la recepción del Quijote en Gran Bretaña comienzan proclamando la excepcional relevancia que la obra de Cervantes alcanzó en los círculos literarios británicos, sobre todo en el siglo XVIII. Cervantes no sólo despertó el interés y causó la admiración de los padres de la novela inglesa, Don Quijote y Sancho se filtraron, además, en la cultura británica hasta llegar a contribuir con sus aventuras a enriquecer los modismos de la lengua inglesa -con el adjetivo quixotic y la expresión to tilt at windmills-. La influencia que el Quijote ejerció en la literatura británica fue notable en los siglos XVII y XIX, y determinante en el XVIII. En la evaluación crítica que escribió sobre The Female Quixote (1752), de Charlotte Lennox, para el Covent Garden Journal, Henry Fielding estimó esta imitación inglesa superior a los méritos del original cervantino, arguyendo que las aventuras de la quijota inglesa gozaban de una estructuración y una credibilidad superiores a las del caballero de la Manchal. Siguiendo a Fielding, y ya en el siglo XX, ciertos críticos ${ }^{2}$ aseveraron que el interés de los británicos por la obra cervantina disminuyó a partir de la publicación de The Female Quixote; mas lo cierto es que los lectores olvidaron pronto la novela de Lennox y que cuando Lennox publica su History of Sir George Warrington; or

* Universidad de Edimburgo.

1. Gerald E. JENSEN (ed.), The Covent Garden Journal (New Haven: Yale University Press, 1915), I, 108-282.

2. Elaine M. Kauvar, "Jane Austen and The Female Quixote", Studies in the Novel, 2 (Summer, 1970), 211-221; y Miriam Rossiter SMALL, "The Female Quixote and Other Quixotic Imitations of the Eighteenth Century", en Charlotte Ramsey Lennox. An Eighteenth-century Lady of Letters (New Haven: Yale University Press, 1935), 64-117.
\end{abstract}


The Political Quixote, en 1797, el Quijote ya había comenzado a inspirar a los intelectos del Romanticismo. Al contrario que la miríada de imitaciones quijotescas, la fama y la admiración que el Quijote despertó en Gran Bretaña perduraron durante esos tres siglos sin apenas verse cercenadas, y adaptándose siempre a las inquietudes de cada una de las tendencias literarias vigentes: en el siglo XVII fue leído como un libro de caballerías, en la primera mitad del XVIII como una parodia de los oligofrénico libros de caballerías, y en la segunda mitad del XVIII, por los románticos del XIX, como una ejemplar exaltación de los valores caballerescos. Estas tres fases de la perspectiva bajo la cual se percibió el Quijote se desprenden del momento literario, pero también de las alusiones que al Quijote se hacen en obras de gran difusión y excepcional calidad literaria, y, muy especialmente, de los prólogos y las notas de las traducciones ${ }^{3}$. Los traductores del Quijote colaboraron en gran medida a la percepción de esta obra en Gran Bretaña de dos modos: (primero) mediante comentarios críticos y desarrollados, fundamentalmente, en introducciones y prólogos, que influyeron la percepción del Weltanschauung cervantino, y (segundo) por medio del trasvase de las características estilísticas del original, lo que condicionó la forma de imitaciones y adaptaciones. En este ensayo pretendo exponer cómo y en qué medida estos traductores condicionaron la influencia del Quijote en la Gran Bretaña de los siglos XVII y XVIII: en las tres fases de percepción del fondo, y en los aspectos formales, esto es, en la estructura narrativa y en el lenguaje.

La primera traducción en lengua inglesa apareció antes de la publicación de la Segunda Parte, bajo el título The History of the valerous and wittie Knight-Errant, Don Quixote of the Mancha, rubricada por Thomas Shelton y publicada en Londres en 1612. Aunque de un entusiasmo muy loable, la traducción de Shelton evidencia la precipitación propia de quien redactó a volapluma y un conocimiento insuficiente de la lengua castellana; el léxico es harto impreciso, sobre todo en la traducción de los parónimos, que siempre toma por palabras cognadas. En 1620, Shelton tradujo la segunda parte, que publicó, junto a la primera, en un volumen que apareció en 1652

3. Traducciones que fueron muchas. El área de la filología inglesa ha producido en España una serie de tesis doctorales (todas ellas inéditas) acerca de las traducciones inglesas de clásicos españoles del Siglo de Oro: la tesis de Julio César SAntoyo sobre el Lazarillo; la de Pedro Guardia sobre Celestina (Universidad de Barcelona, 1971); la de I. Verdaguer sobre el Gızmán (Universidad de Barcelona, 1981); la de José Luis Chamosa sobre la Diana (Universidad de León, 1987). Antes de la tesis doctoral del Quijote (de C. CunChILlos, Universidad de Zaragoza, 1984), las traducciones del clásico cervantino habían sido ya tratadas por varios críticos (que se irán referenciando a lo largo de este ensayo). De las Novelas ejemplares se ocupó en su tesis doctoral Frances LUTTIKHUIzEN (Universidad de Barcelona, 1985). En el volumen De clásicos y traducciones se compilaron capítulos sobre estas traducciones, en la mayoría de los casos de los mismos autores de las tesis doctorales. El capítulo de CuNCHILlos se titula “Traducciones inglesas del Quijote (1612-1800)”. Vid J. C. SANTOYo e I. Verdaguer, De clásicos y traducciones. Clásicos españoles en versiones inglesas: los siglos XVI y XVII (Barcelona: PPU, 1987). (Adviértase que el título del capítulo de Cunchillos contradice el título del libro).

ANALES CERVANTINOS, VOL. XXXVII, PP. 253-265, 2005. ISSN: 0569-9878 
y 1675, convirtiéndose en el único Quijote para todo británico que fuese incapaz de leer castellano. En esta primera traducción hay que advertir dos aspectos: la ya mencionada insuficiencia estilística, y el vacío diacrónico que distanciaba el texto meta del texto origen.

Indudablemente, la imprecisión léxica de Shelton incidió en la equivalencia dinámica de su traducción, pero, sobre todo, es preciso denunciar que se trata de una traducción en diacronía. Mounin ${ }^{4}$, siguiendo a Humbolt, afirma que las diferencias diacrónicas que siempre existen -y que pueden ser de muy diversa índole, v.gr. cronológicas, lingüísticas o culturales- dificultan e imposibilitan la traducción. Aun cuando resulte lícito considerar todas las traducciones como diacrónicas, parece también evidente que algunas lo son más que otras. En cuanto a los tres tipos de contenidos lingüísticos -designación, significado y sentido-, Coseriu ${ }^{5}$ advierte que significado y sentido afectan directamente a la traducción. En el caso del Quijote, el sentido del texto -que es, grosso modo, la parodia- se ha privado de gran parte de su significación en el decurso del tiempo; por ello, los lectores de épocas sucesivas han procurado sentidos que son radicalmente ajenos al principio paródico de esta novela. El vacío diacrónico entre el Quijote de Cervantes y Don Quixote de Shelton no es cronológico, pues a ambos textos los separan apenas siete años, sino cultural. Para entender la diacronía cultural baste con recordar los esfuerzos de Martín de Riquer ${ }^{6}$ por aproximar el verdadero sentido del Quijote al lector del siglo XX, o los más recientes argumentos folklóricos con que Agustín Redondo ${ }^{7}$ asegura que el sentido paródico de esta novela es predominantemente carnavalesco. El vacío diacrónico entre la literatura española y la británica es significativo: para entender el sentido paródico del Quijote, como se esfuerza en explicar Martín de Riquer, han de conocerse las tradiciones pastoril y caballeresca, sobre todo el Amadís. El gusto literario inglés se había nutrido también de la novela pastoril y, especialmente, del ciclo artúrico, pero las numerosas alusiones al Amadís, que, como señala Martín de Riquer, el lector español entendía, le pasan inadvertidas al británico. Por estas razones, el Quijote, que en España fue leído con todas las consecuencias literarias, no despertó en la Inglaterra de Shelton más que consideraciones de tipo ontológico. La ausencia de referencias literarias y el precipitado estilo de Shelton propiciaron que Gran Bretaña viera en Don Quijote sólo a un desequilibrado mental de comportamiento bufonesco. En Gran Bretaña, como en Francia, el Quijote fue entendido como uno más de los libros de caballerías que Cervantes

4. G. Mounin, Los problemas técnicos de la traducción (Madrid: Gredos, 1977), 60 y ss.

5. E. COSERIU, "Creatividad y técnica. Niveles del lenguage", en Lecciones de lingüística general (Madrid: Gredos, 1981), 269 y ss.; y "Lo erróneo y lo acertado en la teoría de la traducción”, en $E l$ hombre y su lenguaje (Madrid: Gredos, 1977), 214 y ss.

6. Martín de RiQuer, Aproximación al Quijote (Madrid: Salvat, 1970).

7. Agustín Redondo, Otra manera de leer el Quijote (Madrid: Castalia, 1997). 
había pretendido parodiar, y los británicos, observa Pritchett $^{8}$, adaptaron a caballero y escudero a la mentalidad de la clase media británica ${ }^{9}$.

La influencia del Quijote en los literatos británicos del siglo XVII se limita, pues, al empleo del personaje protagonista como un demente bufonesco y patético, y se desprende eminentemente de la incapacidad de Shelton de reducir el distanciamiento diacrónico entre el original y su traducción, y de recrear en inglés el complejo y bello estilo cervantino. Los reflejos de esta imagen pueden apreciarse en multitud de textos que aluden a Don Quijote, v.gr. en The Young Gallants Whirliggigg (1629) de Francis Lenton, The Schollers Medley (1614) de Richard Braithwaite y, sobre todo, en las obras de Shirley y de Gayton, a quienes debe atribuirse buena parte de la escarnecedora presentación del caballero de la Mancha. En su Triumph of Peace (1634), Shirley saca a escena a Don Quijote y a Sancho, quienes, dominados por un inexplicable y violento arrebato, embisten contra un honrado caballero inglés. La presentación más negativa de Don Quijote corresponde a Edmund Gayton ${ }^{10}$, quien en sus Pleasant Notes upon Don Quixote califica al protagonista de "sly coward", "unabasted liar", "hypocritical thief" y "meanly-mouthed courtier". Esta imagen jocosa de Don Quijote hizo que los textos que en él se inspiraron fuesen de carácter satírico-burlesco (siendo Hudibras [1663, 1664, 1678] de Samuel Butler el más representativo y afamado de los ejemplos).

John Phillips, sobrino y protegido de John Milton, realizó la segunda traducción del Quijote, publicada en 1687. Philips gozaba de cierta experiencia como traductor: su firma campea en la traducción al inglés de Typhon (1665) de Scarron y es, además, autor de Moronides (1672), un texto de carácter burlesco. Pero en lugar de emplear su experiencia literaria para mejorar el trabajo de Shelton, Philips se limitó a remedar la versión de su predecesor, quizá porque, como en el caso del anterior, y de otros muchos que les seguirían, su castellano no le permitía producir una traducción de calidad. Phillips no colaboró a mejorar la fidelidad de la traducción, como tampoco evitó que la negativa percepción de Don Quijote se alterará en lo más mínimo; por el contrario, su Don Quixote es, apunta Edwin Knowles, "Even more in the Gayton line"11, tendencia ésta que culminaría siete años más tarde con The Comical History of Don Quixote $(1694,1696)$ de Thomas D'Urfey.

8. V. S. PRITCHETT, "Quixote's Translators", en The Working Novelist (Londres: Chatto \& Windus, 1965), 166-171, 166.

9. En su Don Quixote in England: The Aesthetics of Laughter (Baltimore: Johns Hopkins University Press, 1998), Roland PAULSON analiza cómo las dos tendencias políticas del siglo XVII, Tories y Whigs, percibieron el Quijote de modos muy dispares lo que fue especialmente apreciable en las representaciones pictóricas de Don Quijote y Sancho.

10. Vid. Edward M. WILSON, "Edmund Gayton on Don Quixote, Andrés, and Juan Haldudo", Comparative Literature, II (1950), 64-72.

11. Edwin B. KNowles, "Cervantes and English Literature", en Cervantes Across the Centuries, ed. Á. Flores y M.J. Bernadete (New York: Gordian Press, 1969), 277-303, 285. 
La siguiente traducción fue la del Captain John Stevens, publicada en 1700. Stevens era un entusiasta hispanista a quien también se deben traducciones del género picaresco, que agrupó bajo el título The Spanish Libertines: Or the Lives of Justina, the Country Jilt; Celestina, the Bawd of Madrid; and Estevanillo Gonzalez (Londres, 1707; reimpreso en 1709 y 1780)12. Al igual que Phillips, Stevens prescinde de su experiencia literaria, y toma la traducción de Shelton para trabajar a partir de ella. John Stevens, no obstante, incluye su traducción del Quijote apócrifo de Avellaneda en un tercer volumen. La versión de Stevens elude comentarios acerca de la psicología de Don Quijote, y si bien no se manifiesta partidario de las posturas de Gayton, tampoco las replica.

En ese mismo año de 1700 aparece una de las traducciones más difundidas de la historia, la de Peter Motteux y "several hands", la primera en prescindir totalmente de las directrices de Shelton. Motteux se armó de hermenéutica y se permitió anglicanizar el original cervantino, sobre todo el personaje de Sancho Panza. La traducción de Motteux no está exenta de incontables errores: la mala interpretación de modismos y dilogías y la gratuita eliminación e inclusión de adjetivos calificativos la hacen tan censurable como la de Shelton. En Motteux se advierte aún el peso de la noción bufonesca que de Don Quijote se tenía; sin embargo, Motteux reconoce ya un sentido al Quijote: lejos de limitarse a la narración de las locuras de un demente, en su "Account of the Author", con que se prologa el volumen, Motteaux reclama la novela de Cervantes como una crítica a la aristocracia española y presenta a Cervantes como enemigo de la nobleza hispánica: "the wonderful Declesion of the Spanish Bravery and Greatness in this last Century may be attributed very much to [Cervantes's] carrying the Jest too far, by not only ridiculing their Romantic Love and Errantry, but by laughing them also out of their Honour and Courage"13.

No obstante, Motteux no fue el primero en presentar el Quijote como un feroz vejamen dirigido a la aristocracia española. El primer argumento en favor de la iconoclasia del Quijote surge de la pluma del francés Rapin en sus Réflexions sur la poétique d'Aristote et sur les ouvrages des poètes anciens et modernes (1674), vertidas al inglés por Thomas Rymeral en el mismo año de su publicación. Rapin sostenía que Cervantes había escrito el Quijote por despecho y contra el Duque de Lerma, a quien creía enemigo de la clase intelectual. No se puede atribuir a Motteux la iniciación de esta segunda perspectiva de la novela; antes bien, resulta lícito afirmar que sus comentarios en la "Account of the Author" fueron determinantes para que tomara fuerza y se implantara definitivamente en Gran Bretaña. Recuérdese que en aquella época la crítica literaria era escasa, y que al traductor se le atribuía a menudo el

12. Para una relación de las obras de John Stevens vide José Miguel Santamaría, "Captain John Stevens", Livius, I (1992), 211-219.

13. Petter Motreux (trad.), Don Quixote (Nueva York: Random House, 1930), 7. 
más completo conocimiento del texto, por lo que su opinión era, como demuestra el caso de Motteux, decisiva: a partir de la aparición de esta traducción, Gran Bretaña olvida definitivamente al Don Quijote bufón para deleitarse con las aventuras de un Don Quijote, en palabras de Susan Staves, "still ridiculous but noble" 14 .

En 1701, un año después de que apareciera la traducción de Motteux, se publica en Inglaterra la traducción inglesa del Gran Dictionnaire Historique de Louis Moréri, en que el autor reitera la enemistad entre Cervantes y el Duque de Lerma. También entendió Daniel Defoe el Quijote como un ataque a la aristocracia española: en las Memoirs of Captain Carleton (1728) Defoe pinta a un caballero español que explica a Carleton que antes de la publicación del Quijote en España abundaban los esforzados caballeros que trataban de vivir la grandeza de la caballería, pero que la obra de Cervantes logró acabar con tan pretenciosa pomposidad. Esta perspectiva permaneció inalterable hasta mediados del siglo XVIII, y se puede apreciar en los escritos de Steele, Warburton, Shaftesbury, William King y William Collins; después de la primera mitad de la centuria, Horace Walpole afirmaba en una carta a Sir Horace Mann fechada el 10 de julio de 1774, que "Cervantes laughed chivalry out of fashion"15, y Byron proclamaba en Don Juan (XIII, xi) que "Cervantes smiled Spain's chivalry away".

La convicción de que el Quijote había sido concebido para desacreditar a la nobleza española tuvo como inmediato corolario la contemplación paródica de esta novela. Los británicos entendieron que Cervantes pretendió menoscabar a la aristocracia española y, por ende, la literatura nobiliaria por antonomasia, esto es, la novela de caballerías. Como las novelas caballeresca y pastoril se hubiesen convertido en compendios de buenas maneras -sucesoras en buena medida de los de Hebreo o Castiglione en la Edad Media- y sus protagonistas en ideales a imitar por todos los miembros de la nobleza, el sentido que se atribuyó al Quijote no fue sólo social, sino también literario. Y es en la esfera de lo literario donde la segunda de las traducciones de esta segunda fase alcanza toda su relevancia.

En 1742 aparece un nuevo Don Quixote, esta vez de la pluma de Charles Jarvis (o Jervas), cuyos esfuerzos por procurar una equivalencia estilística sorprendieron a sus coetáneos. En su prólogo, Jarvis cuestiona la percepción exclusivamente paródica del Quijote, ya que, arguye, la caballería andante era poco conocida en la Europa de Cervantes, con la excepción de España, por lo que la parodia sólo podía atañer a los españoles. En consecuencia, Jarvis propone "to comprehend the satire, and give light to the beauties of... Don Quixote"16. La traducción de Jarvis dista mucho de la

14. Susan StAves, "Don Quixote in Eighteenth-century England", Comparative Literature, XXIV, 3 (Summer 1972), 193-215, 193.

15. Horace WalPOLE, Letters, ed. P. Toynbee (Londres, 1903-05), IX, 14.

16. Charles JARvis (trad.), The Adventures of Don Quixote (Londres: George Routledge and Sons, n.d.), xxiii. 
anglicanización de la de Motteux, y la rigidez de su fidelidad produce las veces una literalidad que se torna conspicua, especialmente en las frases hechas. La de Jarvis, no obstante, gozó de un éxito y una difusión sin precedentes; y su fidelidad sirvió para que la clase intelectual británica ahondara con no poca emoción en las implicaciones literarias de la supuesta diatriba antiaristocrática que Cervantes llevó a cabo en el Quijote.

El momento literario que Gran Bretaña vivía en el siglo XVIII propició que el sentido de parodia social del Quijote diese paso a la parodia literaria, y que sus aspectos literarios eclipsaran las hipotéticas inquinas del novelista español. La literatura británica de principios del Setecientos se debatía en una pugna entre el idealismo de la prosa heredera de las tradiciones artúrica y pastoril y el realismo iniciado por Defoe y Samuel Richardson ${ }^{17}$. La nueva interpretación del Quijote como parodia literaria fascinó a todos los novelistas que procuraban el modo de establecer una sólida tradición novelesca en Inglaterra, y que veían en Cervantes al autor que había logrado ese mismo objetivo en España. Por esta razón, el Quijote influyó en las técnicas narrativas. Henry Fielding, quien se había servido de Don Quijote a fin de criticar la sociedad inglesa en su drama Don Quixote in England (1733), halló en el Quijote el molde de su ficción novelística ${ }^{18}$.

Esta segunda fase en la apreciación del Quijote finalizó cuando el carácter paródico dejó de resultar relevante para los lectores y los teóricos de la novela, esto es, cuando la prosa inglesa logró superar la moda por la literatura idealista: en 1752 con la publicación de The Female Quixote. The Female Quixote, acaso la imitación más próxima al Quijote de cuantas se hayan escrito, parodia la prosa idealista de los autores franceses del siglo XVII -entonces predilecta de los lectores británicos- con efectos similares a los obtenidos por Cervantes al parodiar los libros de caballerías. La tercera fase, empero, no comenzaría hasta tres años después de la aparición de la novela de Lennox, con la traducción de Tobias Smollett, publicada en 1755.

17. Vid. Ian WatT, The Rise of the Novel (Londres: Chatto \& Windus, 1957); Michael McKeon, The Origins of the English Novel, 1600-1740 (Baltimore y Londres: The Johns Hopkins University Press, 1987).

18. Además de los varios estudios al respecto, véanse los más recientes: Homer Brown, Institutions of the English Novel from Defoe to Scott (Philadelphia: University of Pennsylvania Press, 1997); Brean HAMMOND, "Mid-century Quixotism and the Defence of the Novel", Eighteenth-century Fiction, 10, 3 (April 1998), 1-20; Pedro Javier PARdo García, "Henry Fielding y el román cómico", en La tradición cervantina en la novela inglesa del siglo XVIII (Salamanca: EdicionesUniversidad de Salamanca, 1997), 1452-1791; Pedro Javier PARDo GARCíA, " La otra cara de Cervantes en la novela inglesa del siglo XVIII: Tom Jones y Humphry Clinker", Actas del II Congreso Internacional de la Asociación de Cervantistas (Nápoles: Instituto Universitario Orientale, 1995), 839-854; J. A. G. ARDILA, "Cervantes y la quixotic fiction: sucesión episódica y otros recursos narrativos", Cervantes, XX, 1 (2001), 43-65. 
Al Smollett traductor se le acusa de deberle demasiado a Jarvis y de saber bastante poco castellano. En efecto, Carmine Linsalata ${ }^{19}$ ha demostrado que la traducción de Smollett no es más que una versión de la de Jarvis, cuya redacción quizá pusiera Smollett en manos de un grupo de acólitos. El Don Quixote de Smollett fue publicado en un número de ocasiones menor al de Jarvis -sólo en trece-, pero su interés aquí radica en el prólogo. El escolio con que Smollett prologa su traducción inicia la tercera de las fases de percepción del Quijote, en que se contempla al héroe como paladín de los ideales caballeres$\cos ^{20}$. Afirma Smollett: "We can plainly perceive, from [Cervantes's] own writings, that he himself had a turn for chivalry: his life was a chain of extraordinary adventures, his temper was altogether heroic, and all his actions were, without doubt, influenced by the most romantic notions of honour" 21 . A partir de la evaluación crítica de Smollett, Don Quijote pasó a ser contemplado como un caballero ejemplar. Su carácter paródico continuó inspirando a varios novelistas, mas no con fines literarios, pues no era preciso cuestionar ni erradicar género alguno ${ }^{22}$, sino social, produciendo novelas como Angelica; or Quixote in Petticoats (1758), Fizigigg; or the Modern Quixote (1772), The Spiritual Quixote (1773), etc. Desde el punto de vista literario, Don Quijote se convirtió en, como lo describe Susan Staves, un "idealistic and noble hero"23, y Cervantes, como apunta Smollett en su prólogo, en un genio mártir de la estolidez humana, que hubo de sufrir "all the insults to which he was exposed from the malevolence of mankind"24. A partir de la traducción de Smollett, los británicos descubrieron en la honorabilidad de Don Quijote esa beauty que Jarvis había propuesto que se procurase. Las alusiones al Don Quijote heroico se suceden, siendo la de Henry Brooke en su famoso Fool of Quality (1766) quizá sea la más apasionada de cuantas ofrece este periodo ${ }^{25}$ :

19. Carmine Rocco LinSalata, Smollett's Hoax: "Don Quixote" in English (Stanford: Stanford University Press, 1956).

20. Algunos meses antes de la publicación de la traducción de Smollett habían aparecido los Remarks on the Proposals lately published for a New Translation of Don Quixote (1755) de William Windham. Windham asegura en su ensayo que Cervantes era un ideal de caballero y que en el Quijote pretendía criticar los gustos literarios y no los ideales de la caballería.

21. Tobias SMOLLeTt (trad.), Don Quixote (Londres, 1755), ix.

22. Hasta que Jane Austen considerase necesario parodiar los excesos idealistas de la novela gótica con su Northanger Abbey (1818). Vid. J. A. G. ARDILA, "Cervantes y la quixotic fiction: la parodia de géneros", Anales Cervantinos, XXXIV (1998), 145-168.

23. Susan Staves (op. cit.), 193.

24. Tobias SMOLLETT (op. cit.), xiv.

25. La visión del Don Quijote valiente y generoso ya había sido presentada por Corbyn MORRIS en su Essay Towards Fixing the True Standards of Wit, Humour, Raillery, Satire and Ridicule (Londres, 1744); sin embargo, y aunque anterior a Smollett, Morris no logra imponer la imagen de Don Quijote honorable, quedándose su argumento en un mero comentario anecdótico. Aún en 1750, Samuel Johnson arguía en el número 2 de su Rambler que todos hemos de sentir pena por las fatalidades de Don Quijote -en lugar de la admiración, digna de imitación, que proclama Smollett-. 
How greatly, how gloriously, how divinely superior was our hero of the Mancha! who went about righting of wrongs, and redressing injuries, lifting up the fallen, and pulling down those whom iniquity had exalted. In this his marvellous undertaking, what sufferings, what bruisings, what tramplings of ribs, what poundings of pack staves did his bones not endure?... But, toil was his bed of down, and the house of pain was, to him, a bower of delight, while he consider'd himself as engaged in giving ease, advantage, and happiness to others ${ }^{26}$.

Hoy día, The Fool of Quality es considerado, junto al Sentimental Journey (1768) de Laurence Sterne, el mayor exponente de la escuela sentimental que dominó el panorama novelístico británico de la segunda mitad del siglo XVIII. Una vez más, los literatos británicos hallaron en Don Quijote el psique de sus inquietudes: en este periodo, el héroe de cualidades sentimentales.

La siguiente traducción, realizada por George Kelly y publicada en 1769, no aporta comentario crítico alguno - de hecho se trata de una reimpresión de la de Motteux-. Kelly incluyó la "Account of the Author" de Motteux, en que se presentaba a Cervantes como enemigo de la aristocracia, y que, por tanto, resultaba obsoleta -razón ésta, quizá, por la que no disfrutara de la difusión que las anteriores-. (Kelly sólo añade nueve palabras a la "Account" de Motteux).

La última traducción del siglo XVIII fue realizada por Charles Henry Wilmot y publicada en 1774 . Si Kelly había respetado la visión crítica de Motteux, Wilmot presentó una visión literaria de Don Quijote y de Cervantes análoga a la de Smollett, aunque más exaltada, de lo que se infiere que la perspectiva ennoblecedora que inició Smollett se fue popularizando progresivamente. De Cervantes dice Wilmot que:

On [his] return to his native country, his life and actions were one continued diaplay of virtue, friendship, and minificence; and if, from his taste and education, he was fond of pleasure, still his primary happiness centred in the grand point of DOING GOOD TO HIS FELLOW CREATURES. Purity of principle, and not splendor of situation, was the object which attracted his generous soul. The proud and haughty man he pitied and despised; but the modest and deserving he loved, cherished, and protected. Injured Merit never made her appeal to his humanity without a certainty of relief; nor did Poverty ever approach him, without participating that benevolence with so honourably distinguished and adorned him ${ }^{27}$.

La denominada sentimental fiction de la segunda mitad del siglo XVIII fue definiendo sus peculiaridades hasta dar en el Romanticismo

26. Henry BROOKE, The Fool of Quality (Londres, 1766), 153-154.

27. Charles Henry Wilmot (trad.), Don Quixote (Londres, 1774), iii. 
-este sentimentalismo es, a la verdad, eminentemente prerromántico-. La contemplación de Don Quijote como caballero campeón de egregios ideales se intensificó en el decurso del siglo, paralelamente a la romantización de las letras inglesas. Ciertamente, la aproximación al siglo XIX y el advenimiento del Romanticismo señalan el inicio de la percepción romántica del Quijote. Don Quijote pasó a convertirse entonces en un ideal y, acto seguido, en el héroe del Romanticismo europeo ${ }^{28}$.

No menos interés, tanto traductológico como comparatista, suscita la traducción de los aspectos formales del Quijote. Los traductores del Quijote respetaron la estructura narrativa del original, que influyó en los novelistas británicos del XVIII. Sin embargo, el lenguaje sufrió una importante alteración en la totalidad de las traducciones de los siglos XVII y XVIII, como ha demostrado John Allen. La consecuencia primera de la labor de todos esos traductores -que en ningún caso fueron doctos conocedores de la lengua castellana- es la pérdida irremediable de la riqueza estilística del Quijote. Allen denuncia la incapacidad general de traducir la ironía; en el caso de Motteux afirma que traduce "completely undercutting the edge of parody" 29 , lo que resulta terriblemente significativo si consideramos que es a partir de Motteux cuando se difunde la idea de que Cervantes pretendió parodiar los libros de caballerías por ser el género valuarte de la nobleza española.

La pérdida de la ironía se desprende de todas las fallas que se han apuntado aquí: la incorrecta traducción de parónimos, dilogías y modismos, el descuido en la redacción, la incapacidad -acaso estólida- de los traductores de entender la lengua castellana en toda su dimensión, etc. Asimismo, denuncia Allen que ninguno de los traductores -no sólo estos del XVII y el XVIII, sino cuantos se han aventurado en la experiencia de traducir el Quijote hasta la publicación de su ensayo en 1979- ha sabido establecer una distinción entre los diferentes tipos de lenguaje que aparecen en la novela: ninguno recrea el estilo de los libros de caballerías, por el contrario, confieren a Don Quijote, Micomicona y Trifaldi un estilo contemporáneo al del resto de los personajes, que sólo se distingue por su pomposidad 30 .

Los experimentos con que se ha tratado de denunciar la pérdida de la ironía en las traducciones del Quijote no han resultado inútiles. José María Sbarbi llegó incluso a traducir al castellano el Don Quixote de Smollett para después cotejar el texto resultante con el Quijote de Cervantes. Del experimento

28. Vid. Edward SARmiento, "Wordsworth and Don Quixote", Bulletin of Hispanic Studies, XXXVIII (1961), 113-119; Thomas M. RAYSOR (ed.), Coleridge's Miscellaneous Criticism (Cambridge, Mass., 1936), 102-106; W. U. MCDonALD, Jr., "Scott's Conception of Don Quixote", Midwest Review, I (1959), 37-42; Anthony Close, The Romantic Approach to "Don Quixote" (Cambridge: Cambridge University Press, 1978).

29. John J. Allen, "Traduttori Traditori: Don Quixote in English", Crítica Hispánica, I, 2 (1979), 1-13, 8 .

30. Loc. cit. 
de Sbarbi y del estudio de Allen se infiere que el Quijote no pudo influenciar significativamente el lenguaje de los novelistas británicos del siglo XVIII. Mas la ironía de la prosa cervantina no dejó de impresionar en Gran Bretaña: el reciente estudio de Roland Paulson, Don Quixote in England: The Aesthetics of Laughter ${ }^{31}$, vincula el humor propio y característico de la novela inglesa del XVIII con el Quijote. Tanto el sentido paródico como la estructura narrativa de la novela de Cervantes fascinaron a generaciones de novelistas británicos, quienes, aun desconociendo todo el valor irónico del Quijote, supieron apreciar su fino humor. Resulta imperativo considerar cuál habría sido la influencia que el lenguaje irónico del Quijote habría obrado sobre los novelistas británicos si estos hubiesen tenido acceso a él, y concluir que las traducciones privaron a las letras inglesas de la ironía de cuya ausencia adolecía la sátira ${ }^{32}$.

En su estancia en Barcelona (II, 62), Don Quijote visita una imprenta, donde diserta acerca de las dificultades que el oficio de la traducción entraña:

... el traducir de una lengua en otra, como no sea de las reinas de las lenguas griega y latina es como quien mira los tapices flamencos por el revés; que aunque se ven las figuras, son llenas de hilos que las oscurecen, y no se ven con la lisura y tez de la haz; y el traducir de lenguas fáciles ni arguye ingenio ni elocución, como no le arguye el que traslada ni el que copia un papel de otro papel.

En los varios Don Quixotes que degustaron los lectores británicos del Seiscientos y el Setecientos las figuras con que Cervantes adornó su novela se vislumbran entre los clarooscuros que produce la parca equivalencia dinámica lograda, resultando las percepciones "llenas de hilos que las oscurecen". Sbarbi ${ }^{33}$ afirmó en 1876 que la riqueza estilística del Quijote lo convertía en un texto intraducible, aseveración que despertó una encendida polémica, protagonizada por Sbarbi y Alexander Duffield, autor de una traducción inglesa del Quijote, en torno a la traducibilidad de la novela cervantina. Traducir el Quijote respetando el lenguaje original no es, en absoluto, tan sencillo como "copiar un papel de otro papel"; mas lo cierto es que los estilos de las traduciones que pusieron esta novela al alcance del lector británico de los siglos XVII, XVIII y XIX distaban mucho del original. Las fallas no fueron enmendadas jamás, como demuestra Allen, y las primeras traducciones del siglo XX -de Putnam (Nueva York: Random House, 1959), Cohen (Harmondsworth: Penguin, 1950) y Starkie (Nueva York: New American Library, 1964)- no habrían sino animado aún más la polémica entre Sbarbi y Duffield.

31. Roland PAulson (op. cit.)

32. Sobre la importancia de la sátira en la novela inglesa del siglo XVIII véase Roland PAULSON, Satire and the Novel in Eighteenth-century England (New Haven: Yale Univesity Press, 1967).

33. José María SbaRBI, Refranero español (Madrid: Gómez Fuentenegro, 1876). 
Los traductores del XVII y el XVIII condicionaron la percepción que en Gran Bretaña produjo el Quijote de los modos que se han detallado. Aún en el siglo XX Arthur Effron ${ }^{34}$, que lee el Don Quixote de Cohen, denuncia horrorizado lo paupérrimo de los versos que en la obra aparecen, y cuya carestía de originalidad sólo puede atribuirse a Cohen. También en el siglo XX, los lectores han sufrido de ciertas consejas que resultan sorprendentes y que no hacen sino distorsionar la recepción de la obra: el "Preface" de la edición de Harvard Classics (1909) de la traducción de Shelton mantiene la aseveración de que la Segunda Parte carece de interés alguno, y Cohen ${ }^{35}$ exhorta a los lectores a que eviten la lectura de El curioso impertinente por tediosa ${ }^{36}$. Pero en el caso de los siglos XVII, XVIII y del romanticismo del XIX la labor de los traductores fue determinante: con sus críticas colaboraron decisivamente en la percepción que de Don Quijote y Cervantes se tuvo, y que fue determinante en la transición de idealismo a realismo que marcó el nacimiento de la novela inglesa; y con sus insuficientes traducciones privaron al siglo XVIII de la justa percepción de la ironía cervantina que podría haber provisto a la literatura satírica de nuevas perspectivas.

La cuestión acerca de la intraducibilidad del Quijote poco importa ya: lo cierto es que la autoría de cualesquiera de los Don Quixotes que circularon por Gran Bretaña durante siglos, y que inspiraron a Fielding, Smollett, Sterne, Coleridge, Wordsworth, Dickens, etc., era sólo en parte cervantina, y que los efectos que el Quijote causó en la literatura inglesa se deben en gran parte a los traductores. Miguel de Unamuno jamás leyó a su admirado Kierkegaard a través de traducciones, prefirió armarse de los conocimientos que de las lenguas cognadas del danés tenía para disfrutar de los textos originales. Y Sigmund Freud declaraba orgulloso tras revisar la traducción española de sus Obras completas: "Siendo yo un joven estudiante, el deseo de leer el inmortal Don Quijote en el original cervantino me llevó a aprender, sin maestros, la bella lengua castellana" 37 . Quizá, de haber sido consciente de las fallas de las traducciones, toda la clase intelectual británica habría decidido aprender castellano para degustar el verdadero Quijote $^{38}$.

34. Arthur EFfron, Don Quixote and the Dulcineated World (Austin: University of Texas Press, 1971), 12.

35. J. M. COHEN (trad.), The Adventures of Don Quixote de la Mancha (Harmondsworth: Penguin, 1950), 15.

36. En su traducción de 1885, ORMSBY tilda esta novela intercalada de "nauseous story": John Ormsby (trad.), Don Quixote de la Mancha (Nueva York: Thomas Z. Crowell, 1885), lxi.

37. Sigmund Freud, Los sueños (Madrid: Alianza, 1995), 5.

38. De este parecer era John BowLE, a quien se debe la primera edición crítica del Quijote, quien en su "Letter to Dr. Percy" (publicada como "A Letter to the Reverend Dr. Percy Concerning a New Classical Edition of Historia del Valeroso Cavallero Don Quijote de la Mancha" [Londres: Printed for B. White, Horance's Head, Fleeet St., 1977]) reclamaba "The need to read Cervantes in the original language". Vid. John BowLE, ed. D. Eisenberg, "Letter to Dr. Percy", Cervantes, XXI, 1 (2001), 95145, 133-134:

ANALES CERVANTINOS, VOL. XXXVII, PP. 253-265, 2005. ISSN: 0569-9878 


\section{Resumen}

Este artículo toma como referencia las traducciones inglesas del Quijote publicadas entre 1612 y 1774 a fin de analizar la recepción que la novela cervantina tuvo durante ese periodo en Gran Bretaña. Los prólogos de los traductores ejercieron una gran influjo entre los lectores británicos en las dos centurias en que Cervantes influyó profundamente en el teatro y la novela ingleses. Así, la traducción de Shelton (1612) presentó el Quijote como una sátira, percepción que se mantuvo a lo largo del siglo XVII; la de Motteaux (1700), como una parodia, y la de Smollett (1755), como el modelo de los valores caballerescos.

Palabras clave: Traducciones inglesas, Shelton, Motteaux, Smollett.

\section{Summary}

This paper analyses the English translations of Don Quixote published between 1612 and 1774 in order to examine its reception in Britain during that period. Indeed the prologues written by the translators did had a deep impact on British readers in those two centuries, in which Cervantes became greatly influential upon English playwrights and novelists. Shelton's translation (1612) presented Don Quixote as a piece of satire, a perception that persisted along the 17th century; Motteaux's translation (1700) portrayed it as a parody, and Smollett's version (1755) as the model of knightly morals.

Key words: English translations, Shelton, Motteaux, Smollett.

The true characteristic of ignorance and ill nature is to decry and undervalue the labours of any man where they tend to any useful purpose. I shall apply to my own intentions what your friend $\mathrm{Mr}$ Warton has said of Shakespeare: "If Cervantes is worth reading, he is worth explaining; and the researchers used for so valuable and elegant a purpose, merit their thanks of genius and candour, not the sature of prejudice and ignorance". That his worth reading, is evident from his being read by all with pleasure; they have the smallest share of it who use the dark glass of a translation, those the highest , who enter the spirit of the original. They who are versed in languages cannot but know the possibility of fully comprehending the import and meaning of words and yet find themselves utterly unqualified to express themselves properly in there own. What voltaire says of Hudibras, that it intraduisible, is applicable to almost every original composition of wit, genius, and humour. 